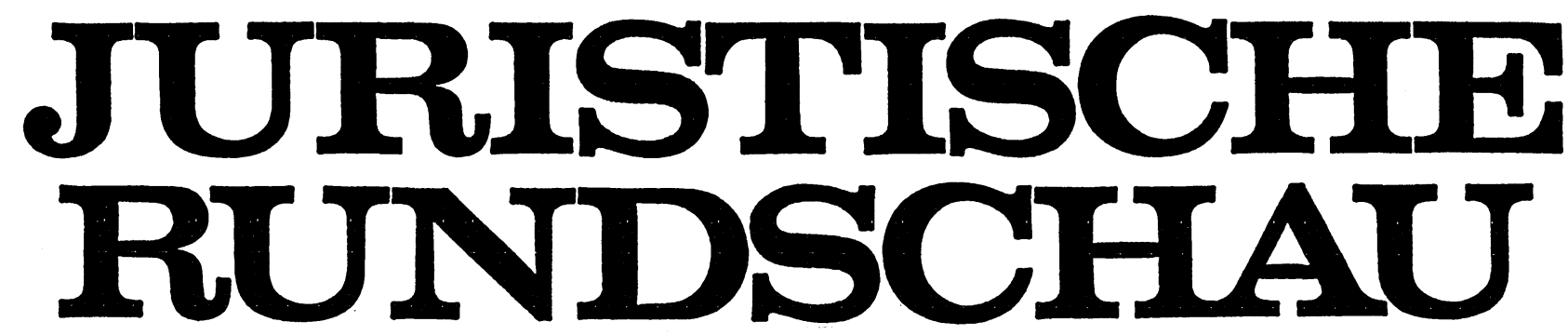

\title{
Schweizerische Rechtsprechung zum deutsch-schweizerischen Anerkennungs- und Vollstreckungsabkommen*
}

\author{
Von Professor Dr. Robert Hauser, Zürich, unter Mitwirkung von lic. iur. Christa Tobler
}

Da die Schweiz dem Übereinkommen über die gerichtliche Zuständigkeit und Vollstreckung gerichtlicher Entscheidungen in Zivil- und Handelssachen nicht beigetreten ist, gilt für die Beziehungen zwischen der Bundesrepublik Deutschland und der Schweizerischen Eidgenossenschaft das Abkommen vom 2.11.1929. Der Aufsatz schildert in einem ersten Teil die Voraussetzungen für die Anwendbarkeit des Staatsvertrages und das schweizerische Vollstreckungsverfahren. Im zweiten, das Hauptgewicht bildenden Teil stellt die Abhandlung die schweizerische Rechtsprechung zum Abkommen dar.

\section{Einleitung}

1. Grundsätzliches über das anwendbare Recht in der Schweiz

Für die Frage der Anerkennung und der Vollstreckung von ausländischen - hier bundesdeutschen - Urteilen in der Schweiz stellt sich zuerst einmal die Frage nach dem maßgeblichen Recht.

a) Kantonales Recht. Gemäß Art. 64 Abs. 3 BV ist das Zivilprozeßrecht Sache des Kantons. Maßgebend ist daher im einzelnen Fall die ZPO des zuständigen Kantons.

In der Folge wird nur die zürcherische ZPO von 1976 berücksichtigt ${ }^{2}$, die die Vollstreckung in den $\$ \$ 300$ ff. regelt.

b) Vorbehalt von Bundesrecht, insbesondere von Staatsverträgen. Staatsverträge gehen als Bundesrecht dem kantonalen Recht vor (derogierende Kraft des Bundesrechtes). Der Bund bzw. das Bundesgericht kann gestützt auf Art. $4 \mathrm{BV}$ in den Bereich der kantonalen Gesetzgebungskompetenz eingreifen, um so die Rechtseinheit zu gewährleisten. Die Kompetenz zum Abschluß von Staatsverträgen erhält der Bund aus Art. 8 BV.

c) Das deutsch-schweizerische Vollstreckungsabkommen. Für Fälle der internationalen Vollstreckung von Urteilen im Verhältnis zwischen der Bundesrepublik Deutschland und der Schweiz ist das Abkommen über die gegenseitige Anerkennung und Vollstreckung von gerichtlichen Entscheidungen und Schiedssprüchen vom 2.11.1929³ maßgebend ${ }^{4}$. Dieses Abkommen gilt nur für die Bundesrepublik Deutschland, nicht aber für die $\mathrm{DDR}^{s}$.

d) Rechtsschutz. Die Verletzung von Staatsverträgen wird höchstinstanzlich in der Schweiz nach Art. 84 lit. c OG auf dem Weg der staatsrechtlichen Beschwerde durch das Bundesgericht geprüft. Mit Bezug auf den Sachverhalt und die staatsvertraglichen Auslegungsfragen steht diesem freie Kognition zu, doch prüft das Gericht nur im Rahmen der in der Beschwerde geltend gemachten Gründe 6 .

\section{Zum Vollstreckungsabkommen (VA)}

a) Zum Zweck. Das VA legt „die Voraussetzungen fest, unter denen die Entscheidungen, die in einem Ver- tragsstaat ergangen sind, im anderen Vertragsstaat anerkannt und vollstreckt werden müssen" "7.

Dabei betrifft die Anerkennung die Frage, welche Rechtswirkung ein ausländischer Entscheid im Inland erzeugt (z.B., ob er die Einrede der rechtskräftig entschiedenen Sache ermöglicht). Die Vollstreckung betrifft die Fälle der ausländischen Leistungsurteile; hier geht es um die Frage der Vollziehung dieser Urteile im Inland ${ }^{8}$.

b) Die nach dem Vollstreckungsabkommen anerkennungsfähigen Entscheide (Art. 1 und 9 VA). Die Frage nach dem sachlichen Geltungsbereich des VA ist die Frage nach den anerkennungsfähigen Entscheiden. Um vom Abkommen erfaßt zu werden, muß ein ausländisches Erkenntnis nach der entschiedenen Materie, der erlassenden Behörde und dem angewendeten Verfahren anerkennungsfähig sein?.

aa) Materie. Erforderlich ist, daß der in Frage stehende Entscheid Zivil- und Handelssachen betrifft; ausgenommen sind jedoch nichtvermögensrechtliche Streitigkeiten, bei denen

\footnotetext{
* Verzeichnis der im Beitrag verwendeten Abkürzungen.

BGE Entscheidungen des Schweizerischen Bundesgerichts, Lausanne $1876 \mathrm{ff}$.

BV Bundesverfassung der Schweizerischen Eidgenossenschaft von 1874 dZPO deutsche Zivilprozeßordnung

OG Bundesgesetz über die Organisation der Bundesrechtspflege von 1943 OR Bundesgesetz betreffend die Ergänzung des Schweizerischen ZivilgeBundesgesetz betreffend die Ergänzung des Schw
setzbuches (5. Teil: Obligationenrecht) von 1911

Pr Praxis des Bundesgerichts, Basel $1912 \mathrm{ff}$.

SchKG BG über Schuldbetreibung und Konkurs von 1889

SJZ Schweizerische Juristenzeitung, Zürich $1904 \mathrm{ff}$

VA Abkommen zwischen der Schweizerischen Eidgenossenschaft und dem Deutschen Reich über die gegenseitige Anerkennung und Vollstreckung von gerichtlichen Entscheidungen und Schiedssprüchen von 1929

ZBJV Zeitschrift des Bernischen Juristenvereins, Bern $1864 \mathrm{ff}$

ZR Blätter für Zürcherische Rechtsprechung, Zürich $1901 \mathrm{ff}$.
}

1 Für die allgemeine schweizerische Literatur vgl. M. Keller/K. Siehr, Allgemeine Lehren des internationalen Privatrechts, 1986, S. 615 ff. mit weiteren Literaturhinweisen.

2 Vgl. die Zusammenstellung bei H. Fritzsche/H.U. Walder, Schuldbetreibung und Konkurs nach schweizerischem Recht, 3. Aufl. 1984, \19 N 28.

3 RGBl. 1930 II 1066 ff.

4 M. Guldener, Internationales und interkantonales Zivilprozeßrecht der Schweiz, 1951, S. 130; R. A. Schütze, Rechtsverfolgung im Ausland, Prozeßführung vor ausländischen Gerichten und Schiedsgerichten, 1986, S. 167.

5 Obergericht Aargau, SJZ 54 (1958) 242.

6 BGE 98 (1972) Ia 532 Erw. 1, Pr 61 (1972) Nr. 150 Erw. 1

7 Guldener (Anm. 4), S. 129.

8 H. Kaufmann, Grundlinien und Entwicklungstendenzen der von der Schweiz geschlossenen zweiseitigen Abkommen über die Anerkennung und Vollstreckung ausländischer Gerichtsentscheidungen in Zivilsachen, ZBJV 112 (1976) $361 \mathrm{ff}$.

Kaumann (Anim. 8y s. 368 . 
ein Drittstaat Partei war (Art. 1 und 3 VA). Erfaßt werden auch Kostenfeststellungsentscheide ${ }^{10}$.

bb) Behörde. Die erkennende Behörde muß nach Art. 1 VA ein bürgerliches Gericht sein, d. h. ein Zivilgericht (ausgeschlossen werden damit von einem Strafgericht erlassene Adhäsionsurteile über Zivilpunkte, ebenso Entscheide von Verwaltungsbehörden wie z.B. der schweizerischen Vormundschaftsbehörde) ${ }^{11}$. Art. 9 VA betreffend Sprüche von Schiedsgerichten ist gegenstandslos, weil heute das Abkommen von New Yorker UN-Schiedsgerichtsübereinkommen vom 10.6.1958 gilt $^{12}$.

cc) Verfabren. Art. 1 VA. verlangt ein Prozeßverfahren, nimmt jedoch Arreste und einstwillige Verfügungen aus.

c) Anerkennung von Entscheiden. Ist ein ausländischer Entscheid anerkennungsfähig, so bleibt für die Frage der Anerkennung zu prüfen, ob er gewisse materielle Kriterien erfüllt. Die wichtigsten dieser Kriterien sind die folgenden:

a) Anerkennungsvoraussetzungen (Art.1-3 VA).

- Die internationale Zuständigkeit, s. hinten II/3,

- die Rechtskraft der Entscheidung in dem Staat, wo sie gefällt wurde, s. hinten II/2 lit. b,

- die rechtzeitige Zustellung der den Prozeß einleitenden Verfügung oder Ladung im Falle von Versäumnisurteilen, s. hinten II/4 lit. d.

bb) Versagungsgrund (Art. 4 VA). Verletzung des ordre public, s. hinten II/4 lit. a und b.

d) Vollstreckung. Die Frage der Vollstreckung stellt sich nur bei ausländischen Leistungsurteilen, die in der Schweiz vollzogen werden müssen. Gemäß Art. 6 VA werden anzuerkennende Entscheide „auf Antrag einer Partei von der zuständigen Behörde dieses Staates für vollstreckbar erklärt". Dies soll in einem möglichst einfachen und schleunigen Verfahren geschehen.

In der Schweiz ist das Zivilprozeßrecht zwar grundsätzlich Sache der Kantone (Art. 64 Abs. 3 BV). Jedoch behält Art. 64 Abs. 1 BV die Gesetzgebung im Betreibungsverfahren und Konkursrecht vor. Auf dem Gebiet der Geldforderung geschieht dies im SchKG ${ }^{13}$. Die Regelung der übrigen $Z$ wangsvollstreckung ordnet durch das kantonale Zivilprozeßrecht, z. B. in $\$ \$ 300 \mathrm{ff}$. der zürcherischen $\mathrm{ZPO}^{14}$. Für die Vollstreckbarerklärung von ausländischen Urteilen ist dementsprechend je nach der Forderung das jeweilige kantonale Prozeßrecht oder das SchKG maßgebend.

aa) Forderungen, die auf Geld oder auf eine Sicherbeitsleistung gerichtet sind. Für die Vollstreckbarerklärung eines ausländischen Urteils, das auf Geld oder auf eine Sicherheitsleistung gerichtet ist, ist der Urteilstitular gemäß Art. 81 Abs. 3 SchKG auf das Rechtsöffnungsverfahren verwiesen. Der Richter entscheidet in diesem Verfahren vorfrageweise über die Vollstreckbarkeit, wobei das Urteil einen Rechtsöffnungstitel bildet ${ }^{15}$. Das bedeutet, daß der Gläubiger kein Vollstreckungsurteil wie nach $\$ 722 \mathrm{f}$. dZPO erlangen muß. Er muß auch kein spezielles Exequaturverfahren anstrengen. Er kann das Urteil im Schuldbetreibungsverfahren (das er zur Durchsetzung seines Anspruches in Gang bringen muß, Art. 38 Abs. 1 SchKG) dem allfälligen Rechtsvorschlag des Schuldners (Opposition des Schuldners gegen die Betreibung, Art. 74 ff. SchKG) direkt entgegensetzen ${ }^{16}$.

Das Urteil bewirkt als Rechtsöffnungstitel die Beseitigung des Rechtsvorschlages und damit die sog. Rechtsöffnung (Art. 80 Abs. 1 SchKG). Damit wird es direkt vollstreckbar (durch Pfändung, Pfandverwertung oder Konkurs, Art. 38 Abs. 2 SchKG). Der Schuldner kann sich zur Wehr setzen, indem er gegenüber dem deutschen
Urteil diejenigen Einwendungen erhebt, die der Staatsvertrag vorsieht (Art. 81 Abs. 3 SchKG).

bb) Forderungen, die auf andersartige Leistungen gerichtet sind (Realexekution). Für die Frage der Vollstreckbarerklärung von ausländischen Urteilen, die auf andersartige Leistungen als Geld oder Sicherheitsleistungen in Geld gerichtet sind, ist wie bereits erwähnt, das kantonale Prozeßrecht maßgebend.

Dieses kann ein besonderes Exequaturverfahren vorsehen. Eine solche Regelung findet sich z. B. in der zürcherischen Zivilprozeßordnung, $\$ 302$ Abs. 2. Als Verfahren wird das sog. Befehlsverfahren ( $\$ 222$ Ziff. 1) vorgeschrieben, welches als summarisches Verfahren ausgestaltet ist. Damit wird der staatsvertraglichen Forderung nach einem möglichst einfachen und schleunigen Verfahren (Art. 6 VA) Rechnung getragen. Die Vollstreckung erfolgt dann nach kantonalem Recht ${ }^{17}$.

\section{Das Verhältnis des Abkommens zum landesrechtlich geregelten Vollstreckungsrecht}

Vollstreckungsfreundlicheres Landesrecht geht dem VA vor, und es ist deshalb unmöglich, den internen schweizerischen Gerichtsstand nicht anzuerkennen, weil der betreffende Gerichtsstand im Staatsvertrag nicht vorgesehen ist ${ }^{18}$. Dazu führt das Obergericht Zürich aus:

„Der Staatsvertrag legt nur fest, unter welchen Voraussetzungen in Deutschland und der Schweiz die Vollstreckung im anderen Staat ergangener Urteile erfolgen müsse, nicht dagegen, wann eine Anerkennung und Vollstreckung erfolgen dürfe. Er will die Vollstreckung erleichtern, nicht erschweren. Ein ausländisches Urteil kann daher im Inland auch Anerkennung finden, wenn die Voraussetzungen dazu auf Grund des Staatsvertrages nicht erfüllt sind; nämlich dann, wenn die Vollstreckung gestützt auf die allgemeinen international-privatrechtlichen Grundsätze des Vollstreckungsstaates begehrt wird.“

Ähnliche Ausführungen finden sich in BGE 94 (1968) II $294 \mathrm{f}$. mit dem ausdrücklichen Vermerk, daß das interne Recht für grenzüberschreitende Streitigkeiten auch Gerichtsstände vorsehen kann, die im deutschschweizerischen Vollstreckungsvertrag nicht enthalten sind.

\section{Die Rechtsprechung zu den einzelnen Bestimmungen des Abkommens}

\section{1. Überblick}

Die hier bearbeiteten Entscheide schweizerischer Gerichte befassen sich mit den Artikeln 1, 2, 4, 5 und 7 des Abkommens. Das Schwergewicht liegt dabei auf den Art. 2 und 4.

10 SJZ 54 (1958) 89; ZR 76 (1977) Nr.107 S. 285 Erw. 6 mit Ausführungen zur Abgrenzung zu Art. 18 und 19 Haager Übereinkunft betr. Zivilprozeßrecht von 1954.

Kaufmann (Anm. 8), S. $366 \mathrm{ff}$

12 R. von Rhein, Zur Anerkennung und Vollstreckung von schweizerischen Gerichtsentscheidungen, Schiedssprüchen und Vergleichen in der Bundesrepublik Deutschland, SJZ 82 (1986) $141 \mathrm{ff}$.

13 K. Amonn, Grundriß des Schuldbetreibungs- und Konkursrechts, 3. Aufl., 1983, § 1 N $13 \mathrm{ff}$.

${ }_{14}$ Zur Realexekution vgl. W.J. Habscheid, Schweizerisches Zivilprozeß- und Gerichtsorganisationsrecht, 1986, \$ 71 N 1284 ff.; O. Vogel, Grundriß des Zivilprozeßrechts, 1984, \63 N $29 \mathrm{ff}$.

15 Guldener (Anm. 4), S. 152; R. Hauser, Ausgewählte Probleme der definitiven Rechtsöffnung, insbesondere bei ausländischen Urteilen, recht 1986, $33 \mathrm{ff}$.

${ }_{16}$ BGE 87 (1961) I 76, 98 (1972) I a 532.

17 Als allgemeine Literatur zum VA vgl. Guldener (Anm. 4), S. 133 ff.; Kaufmann (Anm. 8); R. A. Schütze (Anm. 4), S. 170 ff.; T. S. Stojan, Die Anerkennung und Vollstreckung ausländischer Zivilurteile in Handelssachen, Diss. Zürich 1986.

18 OBergeticht toübich, ZR 68)(1969) skretebibliothek Basel 


\section{Zu Art. 1}

Art. 1 VA lautet: „Die im Prozeßverfahren über vermögensrechtliche Ansprüche ergangenen rechtskräftigen Entscheidungen der bürgerlichen Gerichte des einen Staates werden ohne Unterschied ihrer Benennung (Urteile, Beschlüsse, Vollstrekkungsbefehle), jedoch mit Ausnahme der Arreste und einstweiligen Verfügungen, und ohne Rücksicht auf die Staatsangehörigkeit der an dem Rechtsstreit beteiligten Parteien im Gebiete des andern Staates anerkannt, wenn für die Gerichte des Staates, in dessen Gebiet die Entscheidung gefällt wurde, eine Zuständigkeit nach Maßgabe des Art. 2 begründet war und nicht nach dem Rechte des Staates, in dessen Gebiet die Entscheidung geltend gemacht wird, für dessen Gerichte eine ausschließliche Zuständigkeit besteht."

a) Zum Prozeßverfabren (BGE 86 [1960] I $33 \mathrm{ff}$. $=\operatorname{Pr} 49$ [1960] Nr. 113): In diesem Entscheid geht es um die Frage, ob das deutsche Mabnverfahren ein „Prozeßverfahren" sei.

Gläubiger und Schuldner hatten ursprünglich beide in der BRD Wohnsitz. Dort erwirkte der Gläubiger einen Zahlungsbefehl gegen den Schuldner. Der Zahlungsbefehl wurde für vollstreckbar erklärt, die Vollstreckbarerklärung rechtskräftig. Inzwischen war der Schuldner in die Schweiz gezogen. Es ging nun darum, den deutschen Vollstreckungsbefehl in der Schweiz für vollstreckbar zu erklären. Das lehnte der kantonale Einzelrichter ab mit der Begründung, das Verfahren, in welchem dieser Vollstreckungsbefehl erlassen worden sei, sei mangels genügender Wahrung der Verteidigungsrechte kein Prozeßverfahren i.S. des VA, sondern ein Zwangsvollstrekkungsverfahren. Dazu hielt das Bundesgericht fest:

„Der deutsche Vollstreckungsbefehl ergeht nicht im Zwangsvollstreckungsverfahren ( $\$ \$ 704 \mathrm{ff}$. dZPO), sondern im sog. Mahnverfahren ( $\$ \$ 688 \mathrm{ff}$. dZPO). In diesem Mahnverfahren treten Rechtsträger als Gegner auf, es geht um einen Entscheid über das Recht. Deswegen muß das Mahnverfahren als ein Prozeßverfahren i.S.v. Art. 1 VA qualifiziert werden. Die Verteidigungsrechte des Schuldners bleiben voll gewahrt." Zur Begründung seiner Auffassung verweist das Bundesgericht u. a. auf die Botschaft des Bundesrates zum VA zuhanden des Parlamentes und die Formulierung von Art. 1 VA selbst, wo Vollstreckungsbefehle ausdrücklich als Entscheide genannt werden.

b) Die Rechtskraft des deutschen Entscheides. Das Urteil muß nach Art. 1 und 7 Ziff. 1 VA rechtskräftig sein. $\mathrm{Zu}$ diesem Problemkreis liegt eine Reihe von Entscheiden vor.

aa) Voraussetzungen der Rechtskraft (BGE 82 [1956] I 246 = Pr 46 [1957] Nr. 15): Rechtskräftig ist ein Urteil, wenn es für die Parteien endgültig ist, mit keinem ordentlichen Rechtsmittel mehr angefochten werden kann. Das beurteilt sich für ein deutsches Urteil nach dem deutschen Zivilprozeßrecht. Damit kann vermieden werden, daß im Ausland ein Urteil vollzogen wird, das im Urteilsstaat selber nicht vollstreckbar ist.

bb) Rechtskraft und Vollstreckbarkeit (BGE 82 [1956] I $246 \mathrm{f}$.): Die oben soeben genannte Überlegung führt dazu, daß ein deutsches Urteil in der Schweiz trotz seiner Rechtskraft in der Schweiz nicht vollzogen werden kann, wenn es in Deutschland nicht oder nicht mehr vollzogen werden kann. Eine nach $\$ 769 \mathrm{dZPO}$ nachträglich von einem deutschen Gericht ausgesprochene Suspendierung der Vollstreckung führt deshalb dazu, daß während der Dauer dieser Maßnahme ein Vollzug in der Schweiz ausgeschlossen ist.

c) Zustellung des Entscheids (BGE 96 [1970] I 398, 97 [1971] I 256 ff., 102 [1976] I a 311 ff. = Pr 65 [1976] Nr. 226): Nach $\$ 175$ dZPO wird das Urteil mit der Übergabe an die deutsche Post zugestellt, unbekümmert, ob die Sendung dem im Ausland wohnenden Beklagten zukommt oder nicht. Diese Fiktion hat möglicherweise zur Folge, daß das Urteil in Rechtskraft erwächst, ohne daß der Beklagte die Möglichkeit besitzt, es mit einem Rechtsmittel anzufechten. Das Bundesgericht erblickt darin dann keinen Verstoß gegen den ordre public, wenn der Beklagte im Prozeß auf $\$ 175 \mathrm{dZPO}$ hingewiesen wurde oder das Urteil dem Beklagten durch die schweizerische Post zugestellt wurde.

Diese Rechtsprechung ist auf berechtigte Kritik und Ablehnung gestoßen ${ }^{19}$. Für die Zustellung ist die Haager Übereinkunft über Zivilprozeßrecht von 1954 in Verbindung mit der bilateralen Erklärung zwischen Deutschland und der Schweiz von 1910 über den unmittelbaren Geschäftsverkehr von Behörde zu Behörde maßgebend. Nach diesen Abmachungen sowie nach deutscher und schweizerischer Rechtsauffassung ist die Zustellung unter Vermittlung der Post unzulässig. Eine solche Zustellung vermag deshalb keine Rechtswirkungen zu enthalten ${ }^{20}$. In BGE 105 (1979) I a $312=\operatorname{Pr} 69$ (1980) Nr. 114 äußert denn auch das Bundesgericht Zweifel an der Richtigkeit seiner bisherigen Rechtsprechung.

Zur Rechtskraft äußert sich auch das Obergericht Zürich in ZR 76 (1977) Nr. 107 S. 283 Erw. 4.

Rechtskräftig wird ein deutsches Urteil auch dann, wenn die Zustellung nach $\$ 175 \mathrm{dZPO}$ an den Prozeßbevollmächtigten geschieht, selbst wenn diesem das Mandat gekündigt wurde und ein anderer Anwalt nach $\$ 87$ nicht in Erscheinung getreten ist. Ein Verstoß gegen den schweizerischen ordre public liegt nicht vor. Zur Frage der Eröffnung und Zustellung des in der Schweiz zu vollstreckenden ausländischen (hier iranischen) Urteils hat neuestens BGE 111 (1985) Ia $12 \mathrm{ff} .=\operatorname{Pr} 74$ (1985) Nr.203 Stellung genommen. Danach verlangen der ordre public und Art. 4 BV, daß alle Schuldner in hinreichender Weise Gelegenheit erhielten, vom Urteil Kenntnis zu erlangen, um ein allfälliges Rechtsmittel zu ergreifen, ansonst die Vollstreckung abzulehnen ist. Der Gesuchsteller muß die Rechtskraft und die Zustellung des Urteils an den Gesuchsgegner nachweisen.

d) Fehlende Rechtsmittelbelehrung des zu vollstrekkenden Entscheids (BGE 96 [1970] I 399): Daß das einer ausländischen Partei zugestellte Urteil eine Rechtsmittelbelehrung enthalte, mag wünschbar erscheinen, doch bildet das Fehlen eines solchen Hinweises keinen entscheidenden Grund, das Urteil als offensichtlich der öffentlichen Ordnung zuwiderlaufend zu betrachten. Dies ist schon deshalb angezeigt, weil nicht alle heutigen Prozeßordnungen eine Rechtsmittelbelehrung verlangen.

\section{3. $\mathrm{Zu} \mathrm{Art.} 2$}

Art. 2 VA lautet im wesentlichen: „Die Zuständigkeit der Gerichte des Staates, in dem die Entscheidung gefällt wurde, ist im Sinne des Art. 1 begründet, wenn sie in einer staatsvertraglichen Bestimmung vorgesehen oder eine der folgenden Voraussetzungen erfüllt ist:

1. wenn der Beklagte zur Zeit der Klageerhebung oder zur Zeit der Erlassung der Entscheidung seinen Wohnsitz oder die beklagte juristische Person ihren Sitz in diesem Staate hatte;

2. wenn sich der Beklagte durch eine ausdrückliche Vereinbarung der Zuständigkeit des Gerichts, das die Entscheidung gefällt hat, unterworfen hatte;

19 J.P. Müller, Die staatsrechtliche Rechtsprechung des Bundesgerichts im Jahre 1976, ZBJV 114 (1978) $91 \mathrm{ff}$.; Stojan (Anm. 17) S. $91 \mathrm{ff} . ;$ P. Volken, Internationale Rechtshilfe in Zivilsachen heute und morgen, ZBJV 118 (1982) $451 \mathrm{ff}$.

${ }^{20}$ Analog Cour de justice de Genève zum italienisch-schweizerischen VA von 1933, SJZ 56 (1960) 78 Nr. 28 sowie R. Störner, Urteilsanmerkung, JZ 1987, 45

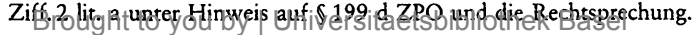


3. wenn der Beklagte sich vorbehaltlos auf den Rechtsstreit eingelassen hatte."

a) Gerichtsstandsvereinbarungen ${ }^{21}$ (BGE 98 [1972] I a 319 ff., Erw. 4 = Pr 61 [1972] Nr. 192): Im Entscheid geht es in erster Linie um die Bedeutung von Art. 59 BV. Dieser garantiert dem zahlungsfähigen Schuldner mit festem Wohnsitz in der Schweiz für obligatorische Forderungen den Wohnsitzgerichtsstand.

Der Schweizer Schuldner hatte beim deutschen Gläubiger wiederholt Waren bestellt, worauf er jeweils Auftragsbestätigungen erhielt. Diese enthielten auf der Vorderseite den Satz: "Wir danken für Ihren Auftrag/Ihre Anfrage, den/die wir zu den umseitigen Verkaufs- und Lieferbedingungen vorgemerkt haben." In den genannten Bedingungen wurde als Gerichtsstand je nach Wahl durch den Gläubiger das Amtsgericht Freudenstatt oder das Landgericht Rottweil genannt. Im Verfahren vor dem Amtsgericht Freudenstatt bestritt jedoch der Schuldner dessen Zuständigkeit; dies tat er auch im Schweizer Betreibungsverfahren. Vor Bundesgericht berief er sich auf Art. 59 BV.

Das Bundesgericht hält fest: „Art. 59 BV bietet keinen Schutz gegen die Vollstreckung eines ausländischen Urteils, wenn die Voraussetzungen, unter denen das Urteil in der Schweiz anerkannt und vollstreckt wird, in einem Staatsvertrag geregelt sind (Zitat mehrerer BGE). Art. 59 BV kann nur insoweit zur Auslegung dieses Staatsvertrages herangezogen werden, als bei dessen Abschluß darauf Rücksicht genommen worden ist.

Für die Prüfung der Frage der Prorogation ist der Schweizer Richter in seiner Entscheidung frei, an die Auffassung des ausländischen Richters nicht gebunden. Es ist zwischen zwei Arten von Gerichtsstandsklauseln zu unterscheiden:

- solchen, die schon im Angebot auf Abschluß des zivilrechtlichen Rechtsgeschäftes enthalten waren und

- solchen, die selbständig sind.

Im vorliegenden Fall liegt eine selbständige Gerichtsstandsklausel vor, da sie erst in den Auftragsbestätigungen enthalten ist. Eine solche Klausel ist nur dann beachtlich, wenn die Gegenpartei eindeutig ihre Annahme ausgesprochen hat. Daran aber fehlt es im vorliegenden Fall, da nicht dargetan worden ist, daß sich die Gegenpartei je zustimmend geäußert hätte."

Es handelt sich hier nicht um ein vereinzeltes Erkenntnis; das Bundesgericht hat seine Auffassung bereits in BGE 84 (1968) I 30 ff. und Pr 61 (1972) Nr. 150 Erw. 3 dargetan. Auch die Basler und Zürcher Rechtsprechung hat diese Grundsätze übernommen; SJZ 60 (1964) 127 f., 64 (1968) 372 ff.

$\mathrm{Ob}$ die Prorogation nach dem deutschen Landesrecht gültig und daher für den Richter verbindlich ist, kann nach Pr 61 (1972) Nr. 150 S. 481 für den schweizerischen Vollstreckungsrichter nicht maßgebend sein. Wichtig ist für ihn, ob von den Parteien eine ausdrückliche Vereinbarung i.S. von Art. 2 Ziff. 2 VA getroffen worden ist, woran - wie gesagt - strenge Anforderungen gestellt werden. Mit Blick auf das schweizerische interne Recht sei erwähnt, daß Zürich $\$ 11 \mathrm{ZPO}$ die Vereinbarung des Gerichtsstandes in einem schriftlichen Vertrag oder in den Satzungen einer juristischen Person verlangt.

b) Einlassung ${ }^{22}$ (BGE 98 [1972] I a $311 \mathrm{ff} .=\operatorname{Pr} 61$ [1972] Nr.150 Erw.4):

Von einer Einlassung kann nicht gesprochen werden, wenn der Beklagte zu Beginn des Prozesses die örtliche Zuständigkeit der deutschen Gerichte bestreitet, die diese Einrede verwerfende Zwischenentscheidung mit allen Rechtsmitteln anficht und sich alsdann vorsichtshalber materiell zur Klage äußert.
BGE 98 (1972) I a 318 Erw. 3 = Pr 61 (1972) Nr. 192: In diesem Entscheid hält das Gericht fest, es sei nicht erforderlich, daß der Beklagte seine Einrede der Unzuständigkeit des deutschen Gerichts bis zum Endentscheid wiederholt. Um eine Einlassung auszuschließen, genügt es unter Umständen, wenn der Schuldner geltend macht, daß er sich in der Schweiz der Anerkennung und Vollstreckung des Urteils widersetzen könne und von diesem Recht Gebrauch machen werde (BGE 96 I 595 ff., 97 I 155/6).

Zur Einlassung äußerte sich das Zürcher Obergericht in ZR 76 (1977) Nr.107 S. 282 Erw.2. Der Beklagte muß vor der Beantwortung der Klage die Zuständigkeit des deutschen Gerichts bestreiten oder darauf hingewiesen haben, daß ihm das Recht zusteht, in der Schweiz der Vollstreckung nach dem VA zu widersprechen.

Diese Bestreitung hat auch dann zu erfolgen, wenn das deutsche Gericht nach seiner lex fori zuständig ist, dieser Gerichtsstand aber in der Schweiz voraussichtlich nicht zur Anerkennung und Vollstreckung führen wird, namentlich weil ein Verstoß gegen die Gerichtsgarantie von Art. $59 \mathrm{BV}$ vorliegt. Denn diese Bestimmung gilt nach BGE 98 (1972) Ia 317 auch für die Gerichtshoheit fremder Staaten, soweit keine abweichenden staatsvertraglichen Abmachungen vorliegen.

c) Der Gerichtsstand des Deliktsortes ${ }^{23}$ (Obergericht Zürich, ZR 68 [1969] Nr. 100): Der Gerichtsstand am ausländischen Begehungsort $(\$ 32 \mathrm{dZPO})$ führt nach Art. 2 VA nicht zur Anerkennung eines dort gefällten Urteils. Auf den ersten Blick mag dies als bedauerliche Lücke erscheinen, speziell wenn man an die aus dem grenzüberschreitenden Straßenverkehr resultierenden Haftpflichtfälle denkt. Doch führt eine nähere Betrachtung zum Ergebnis, daß nach deutschem und schweizerischem internen Recht Urteile des forum delicti commissi jedenfalls bei Straßenverkehrsunfällen gegenseitig anerkannt und vollstreckt werden. Im Entscheid findet sich folgende wörtliche Begründung:

„Abgesehen davon, daß der Vorschrift des Art. 84 Straßenverkehrsgesetz (1958) nach herrschender Lehre und Praxis von jeher international-privatrechtliche Geltung zugesprochen wird, ... ist das forum delicti commissi auch in der deutschen Lehre vom internationalen Privatrecht anerkannt ... Dem entspricht, daß Deutschland für das Herrschaftsgebiet des deutschen Privatrechts diesen Gerichtsstand ebenfalls mit dem Anspruch auf international-privatrechtliche Geltung begründet hat $(\$ 32$ dZPO und als hier in Frage kommende Spezialnorm $\$ 20$ des deutschen Straßenverkehrsgesetzes). Daraus, daß die deutschen Gesetze ebenfalls den Begehungsort als Gerichtsstand für Klagen durch ausländische Motorfahrzeuglenker und -halter geschädigter Inländer kennt, darf geschlossen werden, daß umgekehrt auch Entscheiden gegen deutsche Kraftfahrer am ausländischen Unfallort die Anerkennung und Vollstreckung grundsätzlich nicht versagen wird."

\section{Zu Art. 4}

Art. 4 VA lautet: „Die Anerkennung ist zu versagen, wenn durch die Entscheidung ein Rechtsverhältnis zur Verwirklichung gelangen soll, dem im Gebiete des Staates, wo die Entscheidung geltend gemacht wird, aus Rücksichten der öffentlichen Ordnung oder der Sittlichkeit die Gültigkeit, Verfolgbarkeit oder Klagbarkeit versagt ist.

21 W.J. Habscheid, Parteivereinbarung über die internationale Zuständigkeit nach deutschem und schweizerischem Recht, in: Festschrift für H. Schima, 1969 S. $175 \mathrm{ff}$.

22 J.Schröder, Die Einlassung vor ausländischen Gerichten als Anerkennungsgrund im deutschen Recht, NJW 80, $473 \mathrm{ff}$. (bes. S.474 zur schweizerischen Rechtsprechung).

${ }_{23}$ Näheres dazu bei R. Hauser, Die Geltendmachung von Zivilansprüchen am

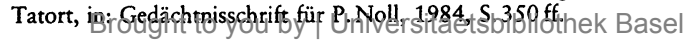


Sie ist ferner zugunsten eines inländischen Beteiligten zu versagen, wenn in der Entscheidung bei Beurteilung seiner Handlungsfähigkeit oder seiner gesetzlichen Vertretung oder bei Beurteilung eines für den Anspruch maßgebenden familienoder erbrechtlichen Verhältnisses oder der dafür maßgebenden Feststellungen des Todes einer Person zu seinem Nachteil andere als die nach dem Rechte des Staates, wo die Entscheidung geltend gemacht wird, anzuwendenden Gesetze zugrunde gelegt sind.

Hat sich der Beklagte auf den Rechtsstreit nicht eingelassen, so ist die Anerkennung zu versagen, wenn die Zustellung der den Rechtsstreit einleitenden Ladung oder Verfügung an den Beklagten oder seinen zur Empfangnahme berechtigten Vertreter nicht rechtzeitig oder lediglich im Wege der öffentlichen Zustellung oder im Auslande auf einem anderen Wege als dem der Rechtshilfe bewirkt worden ist."

\section{a) Zu Art. 4 Abs. 1: ordre public ${ }^{24}$}

aа) BGE 109 (1983) Ib 232 ff. = Pr 73 (1984) Nr. 15: Die Scheidungsklage des deutschen Ehemannes, der mit seiner deutschen Ehefrau in der Schweiz lebte, wurde vom schweizerischen Gericht abgewiesen. Er machte die Klage darauf vor einem deutschen Gericht anhängig. Dieses sprach die Scheidung aus. Zur Zeit des BGE war jedoch die ebenfalls vor dem deutschen Gericht vorgebrachte Forderung der Ehefrau auf Unterhaltsleistungen durch den Ehemann noch nicht endgültig entschieden. Die Ehefrau reichte in der Schweiz ein Gesuch um Eheschutzmaßnahmen ein, das jedoch abgewiesen wurde. Die Begründung war, die Ehegatten seien ja geschieden. Gegen die solcherart erfolgte Anerkennung des deutschen Scheidungsurteils und damit die Abweisung ihres Gesuches reichte die Ehefrau staatsrechtliche Beschwerde ein mit der Begründung, Art. 4 Abs. 1 sei verletzt. Eine erste Verletzung soll darin liegen, daß das deutsche Recht den im schweizerischen Recht geltenden Grundsatz von der Einheit des Scheidungsurteils nicht kennt. Danach müssen auch die finanziellen Folgen der Scheidung im Scheidungsurteil geregelt werden. Eine solche Regelung enthielt das deutsche Scheidungsurteil nicht.

Nach einigen grundsätzlichen Ausführungen zum ordre public hält das Bundesgericht fest:

„Der Grundsatz des innerschweizerischen Rechts bezüglich der Einheit der Scheidung wird vom schweizerischen ordre public nicht erfaßt."

Die zweite Verletzung von VA 4 Abs. 1 soll nach der Ehefrau darin liegen, daß ein deutsches Urteil anerkannt wird, obwohl ein Schweizer Gericht bereits anders entschieden hat. Dazu erklärt das Bundesgericht:

„Grundsätzlich ist von der Autorität einer abgeurteilten Sache auszugehen (Rechtskraft). Dieser Grundsatz gilt jedoch für Scheidungen nicht absolut (Möglichkeit von neuen Gesichtspunkten, neue Lageeinschätzung). Es widerspricht daher nicht dem ordre public, wenn ein ausländisches Gericht eine Scheidung ausspricht, die ein Schweizer Gericht vorher abgelehnt hatte."

Der schweizerische Eheschutzrichter ist an das rechtskräftige Scheidungsurteil gebunden. Er hat keine Möglichkeit, einen allfälligen Widerspruch zwischen dem deutschen und dem schweizerischen Urteil zu überprüfen. Im übrigen widerspricht auch die deutsche Regelung, wonach eine Scheidung auch unabhängig von einem Verschulden eines der Eheleute ausgesprochen werden kann, nicht dem schweizerischen ordre public.

bb) BGE 84 (1958) I 119ff. = Pr 47 (1958) Nr.116: Im Entscheid geht es um den Geltungsbereich des Vorbehaltes des ordre public.
Der Schweizer Schuldner hatte sich gegenüber dem deutschen Gläubiger zu Bürgschaftszahlungen verpflichtet, die er aber nur z. T. erfüllte. Gegenüber der Betreibung in der Schweiz schlug er Recht vor und berief sich auf den schweizerischen ordre public. Das Bundesgericht äußerte sich zuerst allgemein zum ordre public bzw. Art. 4 Abs. 1 VA:

„Art. 4 Abs. 1 soll den Herrschaftsbereich des in der gesamten Rechtsordnung verankerten Vorbehalts der Unvereinbarkeit des fremden mit dem einheimischen Recht auf dem Gebiet der Vollstreckung von Urteilen des anderen Vertragsstaates genauer abgrenzen. Dieser Vorbehalt ermöglicht es, ausländisches Recht ausnahmsweise nicht anzuwenden, wenn es mit der eigenen Rechtsordnung unvereinbar ist und ihrem Sinn und Geist widerspricht. Das einheimische Rechtsgefühl soll nicht in unerträglicher Weise verletzt werden können. Der ordre public kann nicht in allgemein gültiger Weise definiert werden; es muß in jedem Einzelfall entschieden werden (Rechtsgefühl des Richters). Die Vorbehaltsklausel ist einschränkend auszulegen, da sie ja eine Ausnahmebestimmung ist. Bezüglich ausländischer Urteile ist zusätzlich besondere Zurückhaltung geboten."

\section{Zum konkreten Fall führt das Bundesgericht dann aus:}

„Die zur Diskussion stehende Bürgschaft ist nach deutschem Recht abgeschlossen worden. Im Gegensatz zum schweizerischen Recht (Art. 493 Abs. 2 OR) fordert $\$ 766$ BGB keine öffentliche Beurkundung vor dem Notar. Diese wird vom schweizerischen Recht für natürliche Personen bei einem verbürgten Betrag von über $2000 \mathrm{Fr}$. zwingend vorgeschrieben, was aber noch nicht heißt, daß sie deswegen dem ordre public zugehört. Ein Blick auf die Geschichte dieses Erfordernisses der öffentlichen Beurkundung und auf seine Ausgestaltung im geltenden Recht (das Erfordernis ist nur für bestimmte Fälle gegeben) zeigt, daß es nicht vom ordre public erfaßt wird. Seine Weglassung durch das deutsche Recht verstößt daher nicht gegen den schweizerischen ordre public. Somit ist die Bürgschaft gültig und der Anerkennungsversagungsgrund von Art. 4 Abs. 1 VA nicht gegeben."

cc) BGE 85 (1959) I $39 \mathrm{ff} .=\operatorname{Pr} 48$ (1959) Nr.60: In diesem Entscheid geht es wiederum um den Anwendungsbereich des ordre public, hier in bezug auf eine fehlende Vollmacht. Dem schweizerischen Kläger wurden im Prozeß gegen den deutschen Beklagten die Verfahrenskosten auferlegt. Das Problem liegt darin, daß der Prozeß auf Seiten des Klägers und jetzigen Schuldners von einem vollmachtlosen Vertreter geführt worden war. Im schweizerischen Betreibungsverfahren ging es nun darum, ob die Vollstreckung des deutschen Urteils wegen eines Verstoßes gegen den schweizerischen ordre public durch das Fehlen der Vollmacht zu verweigern sei. Dabei war der Beschwerdeführer vor Bundesgericht der Ansicht, der Vorbehalt der öffentlichen Ordnung beziehe sich nur auf den Inhalt der Entscheidung, nicht aber auf das Verfahren. Dazu das Bundesgericht: Der Vorbehalt des ordre public ist seinem Wesen nach ganz allgemeiner Natur. Er bezieht sich auch auf das Verfahren (materieller und formeller ordre public). Eine wörtliche Auslegung von Art. 4 Abs. 1 ist daher abzulehnen. Dabei wird offengelassen, ob Art. 4 Abs. 1 auf alle Verfahrensmängel anzuwenden ist.

Nach einem allgemeinen Grundsatz der schweizerischen Zivilprozeßordnung kann derjenige nicht verurteilt werden, der am Verfahren gar nicht beteiligt war, weil er weder je selber vorgeladen noch richtig vertreten

${ }^{24}$ F. Baur, Einige Bemerkungen zum verfahrensrechtlichen ordre public, in: Festschrift für M. Guldener, 1973 S. $1 \mathrm{ff}$. = Beiträge zur Gerichtsverfassung und zum Zivilprozeßrecht 1983, S.324 ff 
war. Deswegen ist die Vollstreckung eines entsprechenden Urteils zu verweigern. Es verstößt gegen die schweizerische öffentliche Ordnung. Diese Sachlage wird dadurch nicht geändert, daß der Verurteilte die deutschen Rechtsmittelmöglichkeiten gegen das Urteil nicht genutzt hat. Wenn der ausländische Entscheid gegen den ordre public verstößt, darf die Schweizer Prozeßpartei die Vollstreckung abwarten und den Mangel dann geltend machen.

\section{b) Zu Art. 4 Abs. 1 i.V.m. 7 Abs.1:}

\section{Anwaltszwang und Begründung des Versäumnisurteils}

Art. 7 VA lautet im wesentlichen:

„Die Partei, die für eine Entscheidung die Vollstreckbarer-

klärung nachsucht, hat beizubringen:

1. eine vollständige Ausfertigung der Entscheidung; die Rechtskraft der Entscheidung ist, soweit sie sich nicht schon aus der Ausfertigung ergibt, durch öffentliche Urkunden nachzuweisen;

2. die Urschrift oder eine beglaubigte Abschrift der Urkunden, aus denen sich die der Vorschrift des Art. 4 Abs. 3 entsprechende Ladung der nichterschienenen Partei ergibt."

aa) BGE 87 (1961) I 73 ff. = Pr 50 (1961) Nr.79: Der deutsche Gläubiger hatte gegen den Schweizer Schuldner ein deutsches Versäumnisurteil erlangt. Dieses wollte er in der Schweizer Betreibung vollstrecken lassen. Demgegenüber berief sich der Schuldner auf Art. 4 Abs. 1 VA. Die Verletzung des ordre public sah er darin, daß vor dem deutschen Gericht Anwaltszwang bestehe und das Versäumnisurteil keine Begründung enthalte.

Das Bundesgericht hält zunächst fest, daß sich Art. 4 Abs. 1 auch auf das Verfahren bezieht und führt dann aus:

Eine unerträgliche Verletzung des einheimischen Rechtsgefühls liegt nicht schon dann vor, wenn das schweizerische Recht eine Verfahrensvorschrift des ausländischen Rechts nicht kennt. Es muß zusätzlich noch dargetan werden, inwiefern sich diese ausländische Verfahrensvorschrift mit dem einheimischen Rechtsempfinden schlechterdings nicht verträgt.

Der in der Bundesrepublik Deutschland bestehende Anwaltszwang ( $\$ 78 \mathrm{dZPO}$ ) findet keine Entsprechung im schweizerischen Recht. Er bestand jedoch schon lange vor Abschluß des VA. Daß Art. 4 Abs. 3 VA das Recht der persönlichen Prozeßführung nicht als Anforderung an das Verfahren im Urteilsstaat nennt, zeigt, daß der deutsche Anwaltszwang nicht als eine unzulässige Beeinträchtigung der Verteidigung im Prozeß betrachtet werden soll.

Zur fehlenden Begründung im Versäumnisurteil: Art. 7 Abs. 1 verlangt die Beibringung einer vollständigen Ausfertigung des zu vollstreckenden Entscheides. Nach deutschem Recht brauchen Versäumnisurteile, die dem Klagebegehren entsprechen, weder eine Tatbestandsdarstellung noch Entscheidungsgründe zu enthalten (alt $\$ 313$ Abs. $3=313 \mathrm{~b} \mathrm{dZPO}$ ). Die Ausfertigung erfolgt dementsprechend ( $\$ 317$ Abs. 4 dZPO). Eine solche Ausfertigung muß als ,vollständig" anerkannt werden. Anhaltspunkte dafür, daß das VA einen selbständigen Begriff der "vollständigen" Ausfertigung hätte schaffen wollen, liegen nicht vor. Die Bestimmungen der von der Schweiz abgeschlossenen Staatsverträge gelten als Landesrecht mit Gesetzeskraft und können daher nicht gegen die schweizerische öffentliche Ordnung verstoßen.

bb) BGE 103 (1976) Ia 199ff. = Pr66 (1977) $\mathrm{Nr}$. 167: Dieser Fall ist analog zum soeben geschilderten. Wegen der z. T. weitergehenden Begründung des Bundesgerichtes wird er hier dennoch dargestellt. Wiederum geht es um die Frage, ob das Fehlen einer Begründung im
Säumnisurteil einen Verstoß gegen den schweizerischen ordre public darstelle. Das Bundesgericht führte aus:

„Die staatsvertragliche ordre public-Klausel geht als Spezialnorm dem allgemeinen Begriff des ordre public vor. Der Anwendungsbereich des ordre public wird dabei in dem Umfang eingeschränkt, als die Voraussetzungen, unter welchen ein ausländisches Urteil zu vollstrecken ist, im Staatsvertrag näher umschrieben sind. Die Formulierung von Art. 4 Abs. 1 ist eng auszulegen und anzuwenden, bezieht sich jedoch nicht nur auf den materiellen Inhalt eines Urteils, sondern auch auf das Verfahren, in welchem es ergangen ist."

Das Säumnisurteil ist auch ohne Angabe der Entscheidungsgründe „vollständig“ ausgefertigt, da diese Ausfertigungsform den deutschen Verfahrensvorschriften entspricht. Deswegen kann das Fehlen einer Urteilsbegründung nicht als Verstoß gegen den ordre public aufgefaßt werden.

Das Bundesgericht nennt für diese Auffassung historische Gründe und die Auslegung des VA.

\section{c) Rückverweisung}

Zum ordre public vgl. auch vorn II/2 lit. $c+d$.

\section{d) Zu Art. 4 Abs. 3: Säumnisurteile}

Diese Bestimmung befaßt sich mit den Voraussetzungen der Anerkennung und Vollstreckung von Säumnisurteilen.

aa) Begriff (BGE 97 [1971] I 252): Dieser Entscheid folgt der Terminologie des internationalen Zivilprozeßrechts. Nach dieser Auffassung liegt ein Säumnisurteil nicht vor, wenn sich der Beklagte auf den Prozeß eingelassen hat. Die Terminologie der $\$ \$ 330 \mathrm{ff}$. dZPO ist nicht unbedingt maßgebend.

bb) Ladung (BGE 102 [1976] I a 311, 105 [1979] Ib 46 ff. $=\operatorname{Pr} 65$ [1976] Nr. 226, 68 [1979] Nr. 156) (Analog: Obergericht Zürich, ZR 76 [1977] Nr. 107 S. 282 Erw. 3): Hier hält das Bundesgericht fest, daß sich diese Bestimmung nur auf die den Rechtsstreit einleitende Ladung oder Verfügung und nicht auf das Urteil bezieht. Dies ist auch durchaus sinnvoll, soll doch damit nur sichergestellt werden, daß kein Versäumnisurteil in einem Verfahren ergeht und im anderen Vertragsstaat vollstreckt wird, von dem der Beklagte nicht in einer Weise Kenntnis erhalten hat, die ihm die Verteidigung vor dem Prozeßgericht ermöglichte. Für die analoge Anwendung auf das Versäumnisurteil selbst besteht kein Grund. Wäre dies die Meinung der Vertragsstaaten gewesen, so hätten sie dies ausdrücklich so bestimmen müssen.

cc) Nachweis der Ladung (Obergericht Bern, ZBJV 108 [1972] 30 ff.): Nach Art. 7 Ziff. 2 VA ist die Ladung zu belegen. Der schweizerische Rechtsöffnungsrichter, dem der erforderliche Ausweis nicht vorliegt, hat das Gesuch um definitive Rechtsöffnung abzuweisen. Der Gläubiger kann diesen Mangel nicht in appellatorio beheben. Diese Rechtsprechung ist recht streng und verdient keine Zustimmung, wenn nach dem kantonalen Rechtsmittelrecht neue Beweise zulässig sind, wie dies z. B. im Zürcher Zivilprozeß möglich ist ( $\$ 278 \mathrm{ZPO})$.

\section{e) Weitere Fragen}

aa) Rechtshängigkeit (BGE 105 [1979] II $229 \mathrm{ff}$. $=\operatorname{Pr} 69$ [1980] Nr.27): Im Entscheid geht es um die Frage, ob das VA Bestimmungen bezüglich der (Einrede der) Litispendenz enthalte Im sshweizerischen Verfah- 
ren zwischen der Schweizer Schuldnerin und dem deutschen Gläubiger berief sich die Schuldnerin auf die Rechtshängigkeit eines angeblich dieselbe Sache betreffenden Prozesses in der Bundesrepublik Deutschland. Nach Auffassung der Schweizer Gerichte war nicht erwiesen, daß ein solcher Prozeß rechtshängig sei. In dieser Auffassung sah die Schuldnerin eine Verletzung von Bundesrecht.

Das Bundesgericht hält fest, daß die Einrede der Litispendenz im vorliegenden Fall dann nach schweizerischem Bundesrecht $\mathrm{zu}$ prüfen ist, wenn das VA bestimmt, daß der Richter das Eintreten auf eine Sache abzulehnen hat, die schon vorher bei einem Gericht des anderen Vertragsstaates anhängig gemacht worden ist. Eine solche Regelung enthalten die Abkommen mit Österreich und mit Belgien, jedoch dasjenige mit der Bundesrepublik Deutschland nicht.

Die Einrede der Litispendenz ist nach herrschender Lehre $^{25}$ trotzdem zuzulassen, wenn mit einer späteren Anerkennung und Vollstreckung des ausländischen Urteils zu rechnen ist. Die bundesgerichtliche Rechtsprechung geht teils in diese Richtung ${ }^{26}$.

bb) Einrede der abgeurteilten Sache und Vollstrekkung. Anders als z. B. das österreichisch-schweizerische Vollstreckungsabkommen von 1960 enthält sich das VA einer Regelung der Frage, ob ein in der Schweiz in der gleichen Sache ausgesprochenes Urteil der Vollstreckung eines deutschen Urteils entgegensteht. Dafür ist innerstaatliches Recht maßgebend.

Materielle Rechtskraft genießen nach $\$ 191$ Abs. 2 der Zürcher ZPO und Art. 8 VA Erledigungsentscheide, welche auf Grund eines gerichtlichen Vergleiches ergehen. Die Einrede der abgeurteilten Sache in einem neuen in der Schweiz angehobenen Prozeß mit Bezug auf einen vor einem deutschen Gericht abgeschlossenen Vergleich entfällt, wenn dieser Vergleich erfüllt wird, dieser aber später wegen eines Willensmangels angefochten und gegenüber der in der Schweiz niedergelassenen Gegenpartei eine Rückforderungsklage erhoben wird ${ }^{27}$.

cc) Umrechnung in Schweizer Währung. Das Betreibungsbegehren des Gläubigers, welches die Zwangsvollstreckung gegen den Schuldner in der Schweiz einleitet, muß die Forderungssumme ,in gesetzlicher Schweizer Währung " angeben (Art. 67 Abs. 1 Ziff. 3 SchKG). Eine Angabe in einer anderen Währung kann höchstens dann genügen, wenn das (deutsche) Urteil oder der gerichtliche Vergleich zugleich den Umrechnungskurs zahlenmäßig genau festlegt. Ein Urteil oder ein Vergleich, wonach die Umrechnung zu einem erst noch zu ermittelnden Kurs zu erfolgen hat, erlaubt die Fortsetzung der Betreibung nicht $^{28}$. Es ist demnach Sache des Gläubigers, im Betreibungsbegehren den Umrechnungskurs zu bestimmen und summenmäßig auszusetzen. Maßgebend ist der Tag des Betreibungsbegehrens. Die einmal vorgenommene Umrechnung ist für das ganze Zwangsvollstreckungsverfahren verbindlich. Für allfällige dem Gläubiger günstige Kursschwankungen der Fremdwährung muß dieser auf dem Wege einer Nachforderung geltend machen ${ }^{29}$.

\section{Ausblick}

Vor dem eidgenössischen Parlament liegt zur Zeit ein Entwurf für ein schweizerisches IPR-Gesetz in Beratung.

Im Abschnitt 5 des zukünftigen Gesetzes soll die Anerkennung und Vollstreckung ausländischer Entscheide gesamtschweizerisch geregelt werden. An der Anwen- dung und Bedeutung des deutsch-schweizerischen VA wird sich dadurch wenig ändern. Anders verhielte es sich nur, wenn die Schweiz dem EWG-Übereinkommen über die gerichtliche Zuständigkeit und die Vollstreckung gerichtlicher Entscheidungen in Zivil- und Handelssachen vom 27.9.1968 beiträte, was auf längere Sicht hinaus betrachtet nicht als ausgeschlossen zu beurteilen ist.

\section{Zur Sachbeschädigung durch Plakatieren und Beschmieren}

\author{
Von Professor Dr. Friedrich-Christian Schroeder, \\ Regensburg
}

BGHSt. 29, 129 hat das unberechtigte Plakatieren auf technischen Sachen und Gebrauchsgegenständen aus dem Tatbestand der Sachbeschädigung herausgehalten. Der Beitrag erhebt bisher nicht vorgetragene Einwände gegen diese Auffassung.

Mit Beschluß vom 13.11.1979 hat der BGH das unberechtigte Plakatieren auf technischen Sachen und Gebrauchsgegenständen für straflos erklärt ${ }^{1}$. An diesem Beschluß orientiert sich inzwischen wohl oder übel die Rechtsprechung in der Bundesrepublik.

In der Wissenschaft wurde der Beschluß des BGH dagegen überwiegend kritisch beurteilt ${ }^{2}$. Dabei wurde mit Recht auf die unberechtigte Verkürzung des Schutzes des Eigentümers hingewiesen ${ }^{3}$.

Es gibt jedoch noch andere Gründe, die die Entscheidung des BGH unhaltbar erscheinen lassen.

1. Der BGH unterscheidet zwischen dem „Fall, daß die Gebrauchsbestimmung eines Gegenstandes, etwa einer Statue, eines Gemäldes oder eines Baudenkmals, offensichtlich mit seinem ästhetischen Zweck zusammenhängt" und "technischen Sachen und anderen Gebrauchsgegenständen", auch wenn sie nach ästhetischen Gesichtspunkten gestaltet sind und nach ihrer Zweckbestimmung eigene Ansehnlichkeit haben ${ }^{4}$. Bei ersteren soll eine Veränderung der äußeren Erscheinung und Form ausreichen, bei letzteren nicht. Diese Unterscheidung zwischen der „ästhetischen Gebrauchsbestimmung eines Gegenstandes" und der ästhetischen Gestaltung eines Gebrauchsgegenstandes ist mit ihrer Ausgrenzung des „reinen“ Kunstwerks kunsttheoretisch überholt, wenn es sie überhaupt je gegeben hat. Sind gotische Dome und Rokokofassaden nur Gebrauchsgegenstände, die nach ästhetischen Gesichtspunkten gestaltet worden sind?! Ist die Münchner Bavaria ein Kunstwerk, das bestiegen werden kann, oder ein Aussichtsturm in Frau-

\footnotetext{
25 Guldener (Anm. 4) S. 175 ff.; Habscheid (Anm. 14) \31 N 486.

26 BGE 85 (1959) II $85=\operatorname{Pr} 48$ (1959) Nr. 126.

27 Handelsgericht Zürich, SJZ 53 (1957) $254 \mathrm{ff}$.

28 BGE 94 (1968) III 76/77 = Pr 58 (1969) Nr. 21

29 BGE 72 (1946) III $100 \mathrm{ff} .=\operatorname{Pr} 35$ (1946) Nr.173; Amonn (Anm. 13), $\$ 16 \mathrm{~N}$ 10; Fritzsche/Walder (Anm. 2), \$ 16 N 8-10; vgl. dazu im übrigen das Standard-
} werk von B. Kleiner, Internationales Devisen-Schuldrecht, 1985.

1 BGH 29, 129

2 Maiwald JZ 1980, 256 ff.; Gössel JR 1980, 184 ff.; Dölling NJW 1981, 207 Ruthe, Der Normbereich der Sachbeschädigung ( $\$ 303$ StGB), Diss. ErlangenNürnberg, 1980. Für den BGH dagegen H. Katzer, Das unbefugte Plakatieren als Auslegungsproblem der Sachbeschädigung ( $\$ 303$ StGB), Diss. Frankfurt a.M. 1982 und NJW 1981, 2036; Bebm, Sachbeschädigung und Verunstaltung, 1984. 3 Vgl. auch Dreher/Tröndle, StGB, 43. Aufl., 1986, \$303 Rdn. 6; Schönke/ Schröder/Stree, StGB, 22. Aufl. 1985, \$303 Rdn.8; Otto JZ 1985, 28. S. schon früher F.-C.Schroeder JR 1976, $336 \mathrm{ff}$; JZ 1978, $72 \mathrm{f}$.

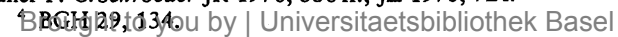

